\title{
ANALYSIS OF COMPLEX TIME FUNCTION OF CONVERTER OUTPUT QUANTITIES USING COMPLEX FOURIER TRANSFORM/SERIES
}

The paper deals with the complex- and discrete Fourier transform which has been considered for both three-and two phase orthogonal voltages and currents of systems. The investigated systems are power electronic converters supplying alternating current motors. Output voltages of them are strongly non-harmonic ones, so they must be pulse-modulated due to requested nearly sinusoidal currents with low total harmonic distortion. Modelling and simulation experiment results of half-bridge matrix converter for both steady-and transient states are given under substitution of the equivalence scheme of the electric motor by resistive-inductive load and back induced voltage. The results worked-out in the paper confirm a very good time-waveform of the phase current and results of analysis can be used for fair power design of the systems.

\section{Nomenclature}

U root-mean-square (RMS) value of phase voltages

$U^{L-L} \quad$ root-mean-square value of line-to-line voltages

$U_{A C} \quad$ magnitude of $A C$ interlink voltage

$U_{D C} \quad$ direct current supply voltage magnitude

$U_{\text {emf }}$ induced count-vise voltage

$A_{v} \quad$ amplitude of $v$-harmonic quantities

$v \quad$ order of harmonics

$f(t) \quad$ time function

$f(t)$ complex time function

$C_{v} \quad$ complex amplitude of v-harmonic

$C^{*} \quad$ complex conjugated amplitude

$f_{s} \quad$ switching frequency

PWM pulse-width-modulation

$f_{1} \quad$ fundamental frequency

$m_{a} \quad$ amplitude modulation ratio

$m_{f} \quad$ frequency modulation ratio

a $\exp (2 \pi / 3)$

$i, k \quad$ indexes

$D C / A C$ voltage sourced inverter

$A C / A C$ direct matrix converter

$T$

time period

\section{Introduction}

Time domain waveforms of electrical quantities can be either continuous or discrete, and they can be either periodic or aperiodic. This defines four types of Fourier transforms: the Fourier series (continuous, periodic), and the Fourier transform (continuous, aperiodic) and discrete versions: the Discrete Fourier Transform - DFT (discrete, periodic), the Discrete Time Fourier Transform (discrete, aperiodic) [1]-[3]. All four members of the Fourier transform family above can be carried out with either real- or complex input data. In spite of complex amplitudes of harmonic components is notation of Fourier series in complex form more compact and easier than pure real expressions. This holds true also for complex Fourier transform which is very close to complex Fourier series [3]. Both of them, Fourier transform and series, operate usually with real time functions [4], [5]. The method of complex conjugated amplitudes has been used for solving of electrical circuits, and electrical machines, too [6], [7].

However, the output quantities of real power electronic converters can be transformed into complex time functions using Park or Clarke transform, respectively, as vectors rotating in complex Gauss plain [8]-[10]. The most advantage of this form of presentation is - in the case of symmetrical sys-tem - that periodicity of the waveforms in the complex plain is $2 m$-times higher than in a real time domain. So, the Fourier analysis, also integral values calculation, can be done more quickly. An-other benefit is possibility of direct use of complex Fourier transform/series because quantity func-tions present complex input data for continuous or digital processing.

\section{Non-harmonic waveforms of power converter output quantities}

Output voltage of a power electronic converter is strongly non-harmonic because of its switching-, pulse nature. The single-

\footnotetext{
* Maria Zaskalicka ${ }^{1}$, Pavel Zaskalicky ${ }^{1}$, Mariana Benova ${ }^{2}$, Mahmud A.R. Abdalmula ${ }^{3}$, Branislav Dobrucky ${ }^{2}$,

${ }^{1}$ Faculty of Electrical Engineering, Technical University of Kosice, Slovakia

${ }^{2}$ Faculty of Electrical Engineering, University of Zilina, Slovakia

${ }^{3}$ University of 7th April, Zawia, Libya
} 
phase voltage inverter with full pulse-width has rectangular waveform output with high content of harmonic components (more than $45 \%$ ). Three-phase inverters produce three line-to-zero (phase) and three line-to-line non-harmonic voltages, Fig. 1.

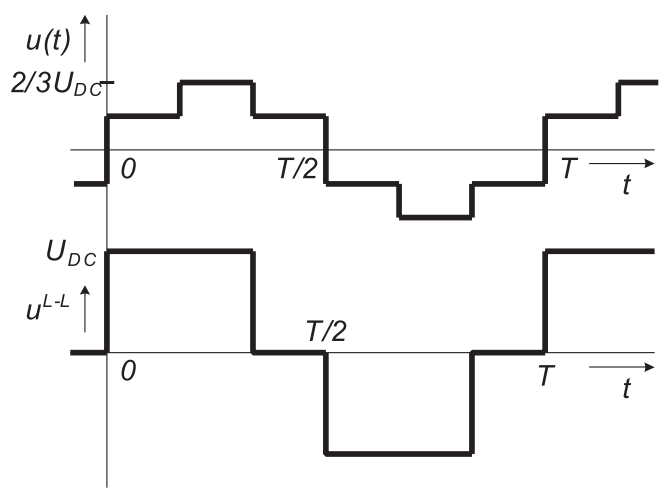

Fig. 1 Output voltages of three-phase inverter with full-width pulses: phase - (a) and line-to line voltage (b)

It is known that the conversion coefficient between phaseand line-to-line harmonic voltages is $\sqrt{3} / 3$, and the magnitude of fundamental harmonic of phase voltage is by $\sqrt{3} / 3$ less than lineto-line voltage.

Based on classical Fourier transform the amplitude of fundamental harmonic of phase voltage can be calculated:

$$
\begin{aligned}
& A_{1}=\frac{8}{T} \int_{0}^{T / 4} f(t) \cdot \sin \omega t d t= \\
& =\frac{4}{\pi} U_{D C}\left\{\int_{0}^{\pi / 3} \frac{1}{3} \cdot \sin \omega t d \omega t+\int_{\pi / 3}^{\pi / 2} \frac{2}{3} \cdot \sin \omega t d \omega t\right\}= \\
& =\frac{2}{\pi} U_{D C}
\end{aligned}
$$

where $U_{D C}$ is supply voltage of the inverter.

Fundamental harmonic of line-to-line voltage (see Fig. 1) is similarly

$$
\begin{aligned}
& A_{1}^{L-L}=\frac{8}{T} \int_{0}^{T / 4} f(t) \cdot \sin \omega t d t= \\
& =\frac{4}{\pi} U_{D C} \int_{\pi / 6}^{\pi / 2} \cdot \sin \omega t d \omega t=\frac{2 \sqrt{3}}{\pi} U_{D C}
\end{aligned}
$$

so, the amplitudes are the same taking in account the relation coefficient.

Also, the total harmonic distortion factor (THD) of both voltages is the same. Let's calculate first the THD of phase voltage

$$
\begin{aligned}
& \sqrt{\frac{\sum U_{v}^{2}}{U_{1}^{2}}}=\sqrt{\frac{U^{2}-U_{1}^{2}}{U_{1}^{2}}}=\sqrt{\left(\frac{U}{U_{1}}\right)-1}= \\
& =\sqrt{\left(\frac{\sqrt{2} / 3}{\sqrt{2} / \pi}\right)-1}=0.3108
\end{aligned}
$$

where $U_{1}$ RMS value of fundamental harmonic of the phase voltage $\left(=A_{1} / \sqrt{2}\right)$,

$$
U=\sqrt{\frac{4}{T} \int_{0}^{T / 6}\left(\frac{1}{3} U_{D C}\right)^{2} d t+\frac{4}{T} \int_{T / 6}^{T / 4}\left(\frac{2}{3} U_{D C}\right)^{2} d t d t}=\frac{\sqrt{2}}{3} U_{D C},
$$

and $U$ is RMS value of phase voltage.

The total harmonic distortion of line-to-line voltage will be the same

$$
\begin{aligned}
& \sqrt{\frac{\sum\left(U_{v}^{L-L}\right)^{2}}{\left(U_{v}^{L-L}\right)^{2}}}=\sqrt{\frac{\left(U^{L-L}\right)^{2}-\left(U_{1}^{L-L}\right)^{2}}{\left(U_{1}^{L-L}\right)^{2}}}= \\
= & \sqrt{\left(\frac{U^{L-L}}{U_{1}^{L-L}}\right)^{2}-1}=\sqrt{\left(\frac{\sqrt{2 / 3}}{\sqrt{6} / \pi}\right)^{2}-1}=0.3108
\end{aligned}
$$

where

$U$ and $U_{1}$ have the same meaning as above, $U^{L-L}=\sqrt{(2 / 3)}$.

Finally, based on total mathematical induction, we can show that both voltages, phase- and line-to-line, comprise the same harmonic components.

Proof \#1:

General relation for $v$-harmonic calculation of line-to-line voltage

$\left|A_{v}^{L-L}\right|=\frac{8}{T} \int_{0}^{T / 4} f(t) \cdot \sin \left(v \frac{2 \pi}{T}\right) d t=$

$=\frac{4}{\pi} U_{D C} \int_{\pi / 6}^{\pi / 2} \sin (v \omega t) d \omega t=$

$=\frac{4}{\pi} \cdot \frac{1}{v} U_{D C}\left[\cos \left(v \frac{\pi}{6}\right)-\cos \left(v \frac{\pi}{2}\right)\right]$

Similarly for phase voltage

$$
\begin{aligned}
& \left|A_{v}\right|=\frac{4}{3 \pi} \cdot \frac{1}{v} U_{D C}\left\{\left[1-\cos \left(v \frac{\pi}{3}\right)\right]+\right. \\
& \left.+2\left[\cos \left(v \frac{\pi}{3}\right)-\cos \left(v \frac{\pi}{2}\right)\right]\right\}
\end{aligned}
$$

From the initial condition for equivalence of the amplitudes

$$
\begin{aligned}
& \left|A_{v}\right|=\frac{\left|A_{v}^{L-L}\right|}{\sqrt{3}} \\
& \frac{1}{3}\left[1+\cos \left(v \frac{\pi}{3}\right)-2 \cos \left(v \frac{\pi}{2}\right)\right]= \\
& =\frac{1}{\sqrt{3}}\left[\cos \left(v \frac{\pi}{6}\right)-\cos \left(v \frac{\pi}{2}\right)\right]
\end{aligned}
$$

For odd $v$-numbers the terms of $[\cos (v \cdot \pi / 2)]$ will be equal to zero. Then

$$
\frac{1}{3}\left[1+\cos \left(v \frac{\pi}{3}\right)\right]=\frac{1}{\sqrt{3}}\left[\cos \left(v \frac{\pi}{6}\right)\right]
$$

After substitution $[1+\cos (v \cdot \pi / 3)]=2[\cos (v \cdot \pi / 6) \cdot \cos (v \cdot \pi / 6)]$ we can obtain 


$$
\cos \left(v \frac{\pi}{6}\right)= \pm \frac{\sqrt{3}}{2} \longrightarrow\left|U_{v}\right|=\frac{1}{\sqrt{3}}\left|U_{v}^{L-L}\right|
$$

Eq. (10) implies that for odd $v$-numbers the condition (7) is fulfilled.

A paradox of different shape of both voltages is possible to explain so that the phase-spectra of the voltages are not the same, they are different.

\section{Using orthogonal output voltages and complex Fourier analysis}

Applying Park/Clarke transform the complex time function of output phase voltage in three-phase system is

$$
\boldsymbol{u}(t)=\frac{2}{3}\left[u_{1}(t)+\mathbf{a} \cdot u_{2}(t)+\mathbf{a}^{2} \cdot u_{3}(t)\right]=u_{\alpha}+j \cdot u_{\beta}
$$

where after adapting

$$
\begin{aligned}
& u_{\alpha}=\frac{1}{3}\left[2 u_{a}(t)-u_{b}(t)-u_{c}(t)\right] \\
& u_{\beta}=\frac{\sqrt{3}}{2}\left[u_{b}(t)-u_{c}(t)\right]
\end{aligned}
$$

It deals with the voltage vectors rotating in Gauss $\alpha, \beta$-plain at an angular speed $\omega$ which can be also non-constant.

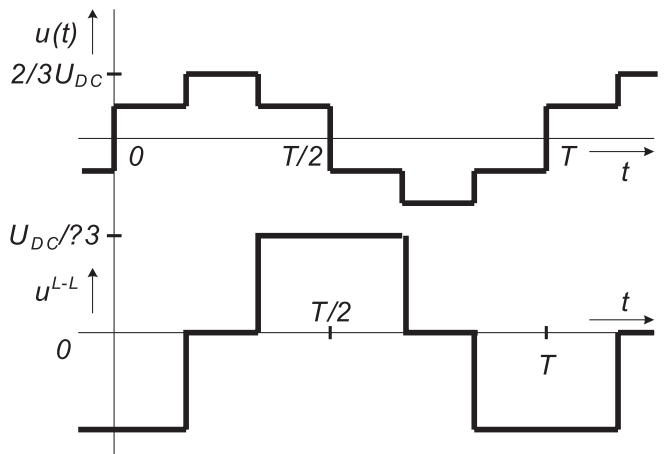

Fig. 2 Orthogonal voltage systems of three-phase inverter with full-width pulses: direct $u_{\alpha}(a)$ - and quadrature voltage $u_{\beta}(b)$

Now, the voltages $u_{\alpha}$ and $u_{\beta}$ create an orthogonal system, and complex Fourier transform can be used.

So, then the complex Fourier transform or/and complex Fourier coefficients can be calculated

$$
\boldsymbol{U}(v \omega t)=\int_{0}^{T} \boldsymbol{u}(t) \cdot \exp (-j \omega t) d t
$$

or, respectively

$$
\boldsymbol{C}_{v}=\frac{1}{T} \int_{0}^{T} \boldsymbol{u}(t) \cdot \exp \left(-j v \frac{2 \pi}{T} t\right) d t
$$

whereby their mutual relation is $\boldsymbol{C}_{v}=\frac{1}{T} \boldsymbol{U}(v \omega t)$ where $\omega=2 \pi / T$.

The discrete Fourier transformation has been used for calculation of individual harmonics coefficients [3]:

$$
\boldsymbol{U}[v]=\frac{1}{N} \sum_{n=0}^{N-1} \boldsymbol{u}[n] \cdot \exp \left(-j v \frac{2 \pi}{N} n\right)
$$

Alternatively, Euler's relation can be used to rewrite the forward transform in a rectangular form:

$$
\boldsymbol{U}[v]=\frac{1}{N} \sum_{n=0}^{N-1} \boldsymbol{u}[n] \cdot(\cos (2 \pi v n / N)-j \cdot \sin (2 \pi v n / N))
$$

Real and imaginary part of $\boldsymbol{U}(v)$ can also be expressed:

$$
\begin{aligned}
& \operatorname{Re}\{U(v)\}=\frac{2}{N} \sum_{n=0}^{N-1} u(n) \cos \left(2 \pi \frac{n v}{N}\right) \\
& \operatorname{Im}\{U(v)\}=\frac{-2}{N} \sum_{n=0}^{N-1} u(n) \sin \left(2 \pi \frac{n v}{N}\right)
\end{aligned}
$$

Based on the above definition the relation for complex Fourier coefficients of complex voltage function yields:

$$
C_{v}=\frac{1}{T} \int_{0}^{T}\left[u_{\alpha}(t)+j u_{\beta}(t)\right] \cdot \exp \left(-j v \frac{2 \pi}{T} t\right) d t
$$

Eq. (16) can be decomposed into two scalar equations for $C_{v}^{\alpha}$ and $C_{v}^{\beta}$, if needed:

$$
\begin{aligned}
& C_{v \alpha}=\frac{2}{T} \int_{0}^{T} u_{\alpha}(t) \cdot \exp \left(-j v \frac{2 \pi}{T} t\right) d t \\
& C_{\nu \beta}=\frac{2}{T} \int_{0}^{T} u_{\beta}(t) \cdot \exp \left(-j v \frac{2 \pi}{T} t\right) d t
\end{aligned}
$$

Such a Fourier series is developed on the system of orthogonal functions $\exp (j \cdot n .2 \pi \cdot t / T), n=0, \pm 1, \pm 2 \ldots$, for which the integral

$$
\int_{0}^{T} \exp \left(-j n \frac{2 \pi}{T} t\right) \exp \left(-j m \frac{2 \pi}{T} t\right) d t
$$

is equal to 0 for $m \neq-n$, and equal to $T$ for $m=-n$.

The system of voltages is ortho-normal, too. Since $u_{\alpha}$ voltage will contain sin-terms only, the second one $u_{\beta}$ cos-terms.

Orthogonal voltage system for a two phase converter system consisting of two single-phase matrix converters

The orthogonal two-phase converter system comprises two single-phase converters. Supposing bridge connection single-phase matrix converter [7], [14] then for its output voltage can be applied pulse-width modulation (PWM). The second voltage of the 2-phase orthogonal system, generated by a second single-phase bridge matrix converter has the same waveform but it is shifted by 90 degrees (or $\pi / 2$ respectively) to the right. Thus the complex Fourier transform and analysis described above can be used. However, based on proof \#1 the amplitude spectra $C_{v \alpha}$ and $C_{v \beta}$ of $u_{\alpha}$ and $u_{\beta}$ will be the same, so it is enough to calculate only one of them. 
The sinusoidal PWM is mature technology which can be classified into Natural sampled (analogue sinetriangle), regular sampled (digital sine-triangle) and space vector modulation. The natural Sampled and regular Sampled also can be divided into two-level (bipolar) or three-level (unipolar) PWM [5], [11]. There are two major concerns for generating sinusoidal PWM. The first is to minimize the creation of low order harmonics in the output voltage. The second is the mitigation of switching frequency harmonics. Both these concerns are controlled by the shape of the PWM pattern which should be controlled to minimize the generation of switching harmonics and maximum the harmonic cancellation between the line-line voltages.

The unipolar PWM was chosen as the best PWM for voltage control of single-phase inverters considering the output voltage harmonic spectrum. Normal unipolar sinusoidal PWM is shown in Fig. 3.

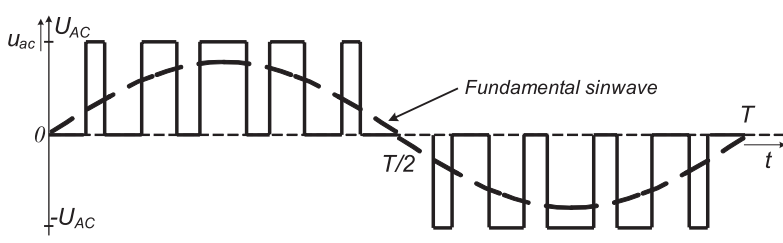

Fig. 3 Output voltages of single-phase bridge matrix converter with PWM technique

It can be observed that the area of each pulse corresponds approximately to the area under the sine-wave between the adjacent mid-points of the off-periods. The pulse-width modulated wave has much lower low/order harmonic content than the other waveforms. Generally the synchronous modulation is used. In synchronous modulation the modulation frequency is an integer multiple of the frequency of reference sine-wave [5], [13]. The turn on $(\alpha)$ and turn off $(\beta)$ angles are calculated by the discreet substitution of the reference sine-wave.

Both amplitude- and frequency modulation ratios $m_{a}$ and $m_{f}$ are defined as:

$$
m_{a}=\frac{U_{1 m}}{U_{A C}} ; \quad m_{f}=\frac{f_{s}}{f_{1}},
$$

where

$U_{1 m}$ is reference amplitude of fundamental harmonic,

$U_{A C}$ magnitude of supply voltage,

$f_{s} \quad$ switching frequency,

$f_{1} \quad$ fundamental frequency.

So, the peak amplitude of the fundamental harmonic component (equal to reference voltage) is $m_{a}$ times $U$, and varies linearly with $m_{a}$ (providing $m_{a} \geq 1$ ). If the frequency modulation ratio $m_{f}$ is sufficiently great, the difference between real values and discrete values is negligible.

The converter's output voltage (Fig. 3) can be mathematically expressed as a Fourier series of the form [5], [12], [13].

$$
u(t)=\sum_{v=1}^{\infty} \frac{2 U}{v \pi} \sum_{k=1}^{\left(m_{f} / 4\right)-1}\left(\sin 2 v \alpha_{k}-\sin 2 v \beta_{k}\right) \sin (v \omega t)
$$

where $\alpha_{k}$ and $\beta_{k}$ - are turn-on and turn-off angles calculated for each $k$-interval by [13]:

$$
\begin{aligned}
& \alpha_{k}=\frac{2 k \pi}{m_{f}}-m_{a} \frac{\pi}{m_{f}} \sin \frac{2 k \pi}{m_{f}} \\
& \beta_{k}=\frac{2 k \pi}{m_{f}}+m_{a} \frac{\pi}{m_{f}} \sin \frac{2 k \pi}{m_{f}}
\end{aligned}
$$

Calculated output voltage for $m_{a}=1, m_{f}=18$ and finite number of harmonics is depicted in Fig. 4 .

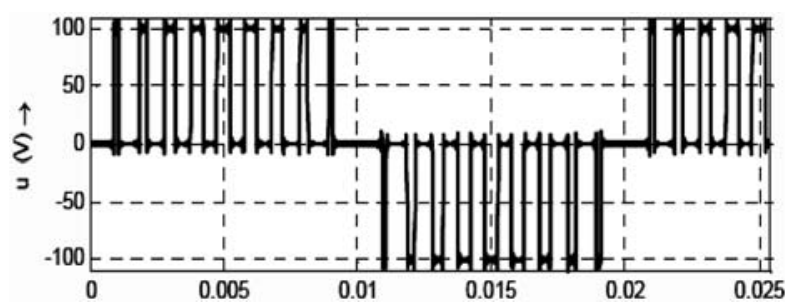

Fig. 4 Output voltages of single-phase bridge matrix converter with PWM technique

\section{Considering single-phase inverter and unipolar PWM with even} $\boldsymbol{m}_{f}$

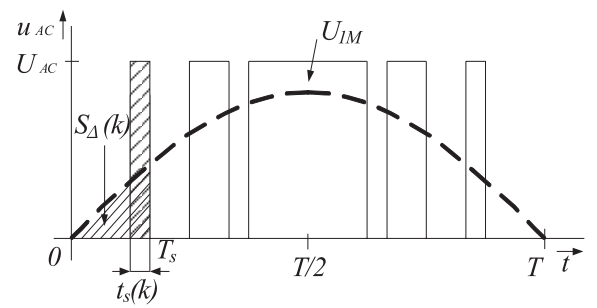

a)

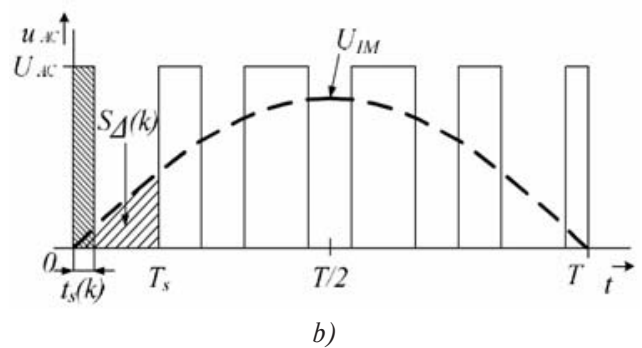

Fig. 5 Output voltages of single-phase bridge matrix converter with even $m_{f} a$ ) or $b$ ) 
Then total voltage time waveform will be:

$$
u(t)=\sum_{v=1}^{\infty} \frac{4 \cdot U_{D C}}{v \cdot \pi} \sum_{k=0}^{\left(m_{f} / 4\right)-1}\left[\cos \left(v \cdot k \cdot \frac{2 \pi}{m_{f}}\right)-\cos \left(v \cdot k \cdot \frac{2 \pi}{m_{f}}+v \cdot \frac{2 \pi}{T} \cdot t_{s}(k)\right)\right] \cdot \sin (v \omega t)
$$

where $t_{s}(k)$ is the switching instant at $k$-interval:

$$
t_{s}(k)=\frac{1}{U_{D C}} \cdot S_{\Delta}(k)=\frac{m_{a} \cdot m_{f}}{2 \pi}\left[U_{m} \cdot \frac{m_{f}}{2 \pi} \cdot\left[\cos \left(\frac{2 \pi}{m_{f}} \cdot k\right)-\cos \left(\frac{2 \pi}{m_{f}} \cdot k+1\right)\right]\right]
$$

and $S_{\Delta}(k)$ is the area under sinewave during $k$-switched interval:

$$
S_{\Delta}(k)=U_{m} \cdot \frac{m_{f}}{2 \pi} \cdot\left[\cos \left(\frac{2 \pi}{m_{f}} \cdot k\right)-\cos \left(\frac{2 \pi}{m_{f}} \cdot(k+1)\right)\right]
$$

\section{Complex Fourier analysis of the voltage of AC/AC half-bridge matrix converter system}

Due to rather high number of power semiconductor switches of both converters (totally 8), the half-bridge connection of the matrix converter is a better solution. The equivalent circuit diagram of one half-bridge single phase converter (one of two-phase orthogonal systems) is depicted in Fig. 6. Since the voltages of the matrix converter system are orthogonal, the second phase converter is the same and its voltage is shifted by 90 degree. Due to half-bridge connection the bipolar PWM should be used.

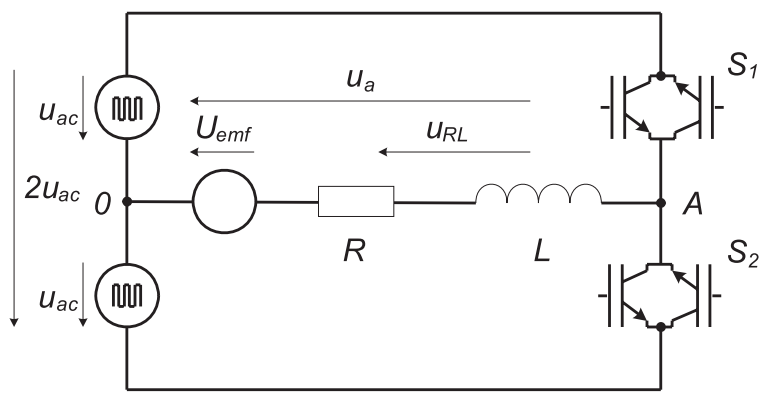

Fig. 6 Circuit diagram of single-phase half-bridge matrix converter

Contrary to the bridge matrix converter the half-bridge connection doesn't provide unipolar PWM control, so the bipolar pulse switching technique should be used. Based on bipolar PWM control the output orthogonal voltages will be presented in Figs. $8 \mathrm{a}$ and $8 \mathrm{~b}$. This type of control technique is more complicated than unipolar type.

Considering bipolar PWM with switching frequency equal to odd multiply of fundamental frequency.

Consequently, the harmonics in the converter output voltage waveform appears as sidebands, centered on the switching frequency $f_{s}$ and its multiples, that is, around harmonics $m_{f}, 2 m_{f}$, $3 m_{f}$, and so on. This general pattern holds true for all $m$ a smaller than (or equal to) 1 . For a frequency modulation ratio $m \mathrm{f}>=9$ (which is our case), the harmonic amplitudes are almost indepen- dent on $m f$, though $m f$ defines the frequencies at which they occur. Theoretically, the frequencies at which voltage harmonics occur can be defined as

$$
f_{v}=\left(x \cdot m_{f} \pm y\right) \cdot f_{1},
$$

that is, the harmonic order $v$ corresponds to the $y$-th sideband of the $x$-times the frequency modulation ratio $m_{f}$

$$
v=\left(x \cdot m_{f} \pm y\right) \cdot f_{1},
$$

where the fundamental harmonic frequency corresponds to $v=1$. For odd values of $x$, the harmonics exist only for even value of $y$, and opposite, for even values of $x$, the harmonics exist only for odd value of $y$.

Choosing the frequency modulation ratio $\mathrm{mf}$ as odd integer results in an odd symmetry $[u(-t)=-u(t)]$ as well as half-wave symmetry $\left[u(-t)=-u\left(t+T_{s} / 2\right)\right]$ with the time origin shown in Figs. 3 or 4 . Therefore, only odd harmonics are present and the even harmonics disappear from the wave form of $u$ a. Moreover, only the coefficients of the sine series in Fourier analysis are finite; those for the cosine series are zero.

The harmonic spectrum is plotted in Fig. 7, which is plotted for $m_{f}=39$.

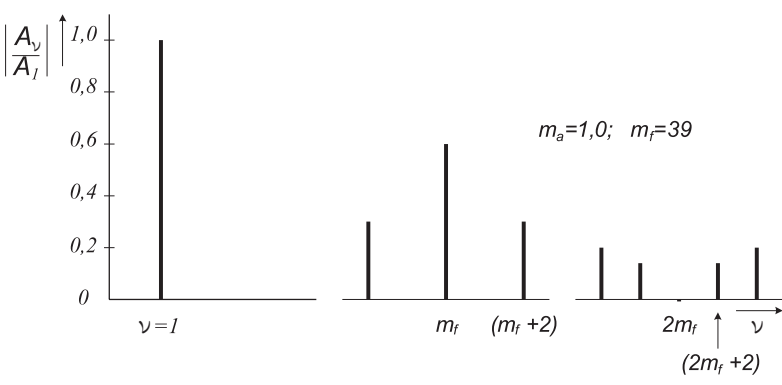

Fig. 7 Harmonic amplitude spectrum of bipolar PWM with odd $m_{f}$

For the parameters (the same as in [11] to be compared):

$Z_{x} U_{D C}=300 \mathrm{~V} \quad$ - input voltage,

$f_{I N}=f_{S}=39 \mathrm{kHz} \quad$ - switching frequency,

$f_{\text {OUT }}=50 \mathrm{~Hz} \quad$ - fundamental output frequency,

$m_{a}=1 ; m_{f}=39 \quad$ - amplitude and frequency ratios, 
the amplitudes of the first 30 voltage harmonics (by $165^{\text {th }}$-harmonic) were calculated:

$A_{1}=150 . m_{a}=150 \mathrm{~V} ; A_{39}=90.16 \mathrm{~V} ; A_{39-2}=A_{39+2}=47.70 \mathrm{~V} ;$

$A_{39-4}=A_{39+4}=2.70 \mathrm{~V}$;

$A_{78-1}=A_{78+1}=27.15 \mathrm{~V} ; A_{78-3}=A_{78+3}=31.80 \mathrm{~V}$;

$A_{78-5}=\mathrm{A}_{78+5}=4.95 \mathrm{~V} ; \mathrm{A}_{117}=16.95 \mathrm{~V}$;

$A_{117-2}=A_{117+2}=9.30 \mathrm{~V} ; A_{117-4}=A_{117+4}=23.55 \mathrm{~V}$;

$A_{117-6}=A_{117+6}=6.60 \mathrm{~V} ; A_{156-1}=A_{156+1}=10.20 \mathrm{~V}$;

$A_{156-3}=A_{156+3}=1.35 \mathrm{~V} ; A_{156-5}=A_{156+5}=17.85 \mathrm{~V}$;

$A_{156-7}=A_{156+7}=7.50 \mathrm{~V}$;

Note: The carried-out results are identical with those given in [11] for DC/AC inverter with bipolar sinusoidal PWM.

Considering bipolar PWM with switching frequency equal to even multiply of fundamental frequency.

The orthogonal voltages with bipolar PWM control are depicted in Figs. 8a, b.

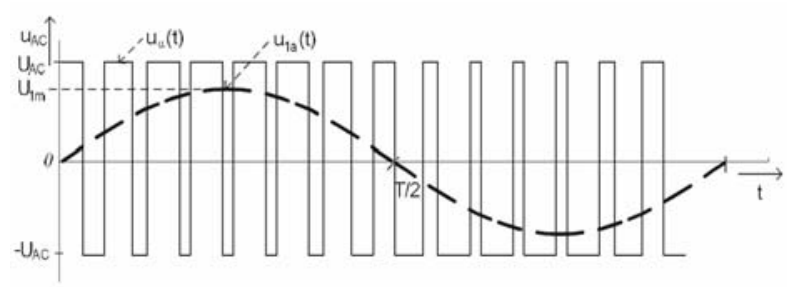

a)

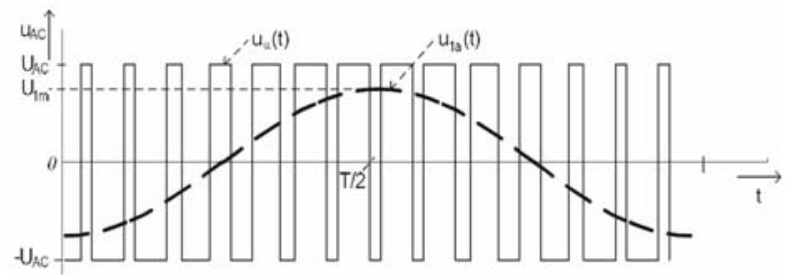

b)

Fig. 8 Output orthogonal voltages of the half-bridge matrix converter system with bipolar pulse-width-modulation: direct (a) and quadrature one $(b)$

It deals with sinusoidal bipolar pulse-width-modulation contrary to unipolar regular PWM [5], [11]. Switching-pulse-width can be determined based on equivalence of the average values of reference waveform and resulting average value of positive and negative switching pulses area during a switching period (see Figs. $5 \mathrm{a}, \mathrm{b}$ and $8 \mathrm{a}, \mathrm{b}$ ).

Then, the total voltage time waveform will be: where the switching instant is equal to:

$$
t_{s}(k)=\frac{1}{2 U_{D C}} \cdot S_{\Delta}(k)+\frac{T_{s}}{2}
$$

and the area under sinewave is the same as using unipolar PWM (see Eq. 21).

\section{Current harmonics investigation under resistive- inductive load with $\mathrm{Uemf}$}

Current time-waveforms for a fundamental harmonic component in steady-state $i_{S 1}(t)$ is [12]:

$$
\begin{aligned}
& i_{s 1}(t)=\frac{A_{1}-U_{e m f}}{Z_{1}} \cdot \sin \left(\omega t-\varphi_{1}\right)= \\
& =\frac{U}{Z_{1}} C_{I}(1) \cdot \sin \left(\omega t-\varphi_{1}\right)
\end{aligned}
$$

Current time-waveforms for higher-harmonic components in steady-state $i_{S v}(t)$ are given [12]

$$
\begin{aligned}
& i_{S v}(t)=\frac{A_{v}}{Z_{v}} \cdot \sin \left(v \omega t-\varphi_{v}\right)= \\
& =\frac{U}{Z_{v}} C_{I}(v) \cdot \sin \left(v \omega t-\varphi_{v}\right)=I_{v} \cdot \sin \left(v \omega t-\varphi_{v}\right)
\end{aligned}
$$

where:

$A_{v}=A_{1} \cdot C_{v}(v)$ - amplitude of $v$-harmonic voltage component extracted from Eq, (23),

$A_{1}=m_{a} \cdot U_{A C}$ - amplitude of 1 . harmonic voltage component, $U_{\text {emf }} \quad-$ counter-voltage (of electromagnetic force)

$\left|Z_{v}\right|=Z_{v}=\sqrt{R^{2}+(v \cdot \omega \cdot L)^{2}}$ - module of complex impedance of resistive-inductive load

$\varphi_{v}=\arctan (v \cdot \omega \cdot L / R)-\operatorname{argument}$ of complex impedance of resistive-inductive load

$C_{I}(v) \quad$ - Fourier coefficient of $v$-harmonic current component,

$I_{v} \quad$ - amplitude of $v$-harmonic current component.

Harmonic current components can be computed similarly using above methodology and work [12]. The accurate calculation of $U_{\text {emf }}$ can be obtained by using the motor circle diagram. The total current in steady-state will be summarisation of single harmonics.

The Fourier analysis can be used also for investigation of behaviour of the system in transient state. The total current of $v$-harmonic component $i_{v}$ will be summarisation of current in steadystate $i_{S v}$ and current in the transient phenomenon $i_{T v}$

$$
\begin{aligned}
& i_{v}(t)=i_{S v}(t)+i_{T v}(t)=\frac{A_{v}}{Z_{v}} \cdot \sin \left(v \omega t-\varphi_{v}\right)+ \\
& +\frac{A_{v}}{Z_{v}} \cdot \sin \varphi_{v} \cdot \exp (-t / \tau)
\end{aligned}
$$

$$
\begin{aligned}
& u(t)=\sum_{v=1}^{\infty} \frac{4 \cdot U_{D C}}{v \cdot \pi} \sum_{k=0}^{\left(m_{f} / 4\right)-1}\left\{\left[\cos \left(v \cdot k \cdot \frac{2 \pi}{m_{f}}\right)-\cos \left(v \cdot k \cdot \frac{2 \pi}{m_{f}}+v \cdot \omega \cdot t_{s}(k)\right)\right]-\right. \\
& \left.-\left[\cos \left(v \cdot(k+1) \cdot \frac{2 \pi}{m_{f}}-v \cdot \omega \cdot t_{s}(k)\right)-\cos \left(v \cdot k \cdot \frac{2 \pi}{m_{f}}\right)\right]\right\} \cdot \sin (v \cdot \omega \cdot t)
\end{aligned}
$$


where $i_{v} \quad$ is total current waveform

$i_{S v} \quad$ steady-state component of total current

$i_{T v} \quad$ transient component of total current

$\tau=R / L-$ time constant of resistive-inductance load.

Total current as well as both components should be calculated for each harmonic.

\section{PC simulation in MatLab programming environment}

The MatLab programming environment was used for simulation. Simulation experiments were done for the parameters: $R=$ $=0.1275 \mathrm{Ohm}, L=21.6 \mathrm{mH}, U_{A C}=12 \mathrm{~V}, f=100 \mathrm{~Hz}$ at $m_{a}=1$, $m_{f}=100, U_{\text {emf }}=0.1-0.9$ of $A_{1}$, time increment $\Delta t=0.5 \mu \mathrm{s}$. b.

The graphic interpretation of steady-state is shown in Figs. 9a,

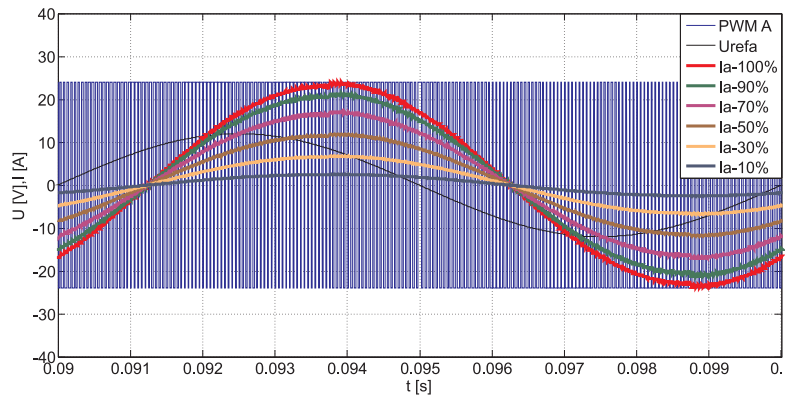

a)

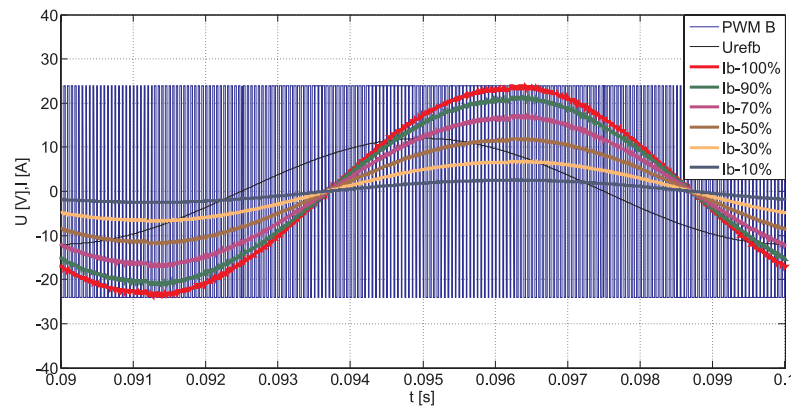

b)

Fig. 9 Output orthogonal voltages of the half-bridge matrix converter system with bipolar pulse-width-modulation and $m_{a}=0.2,0.4,0.6,0.8$, direct phase-(a) and quadrature phase voltage (b)
Time wave-forms of currents for transient-state and various time constants are depicted in Fig. 10.

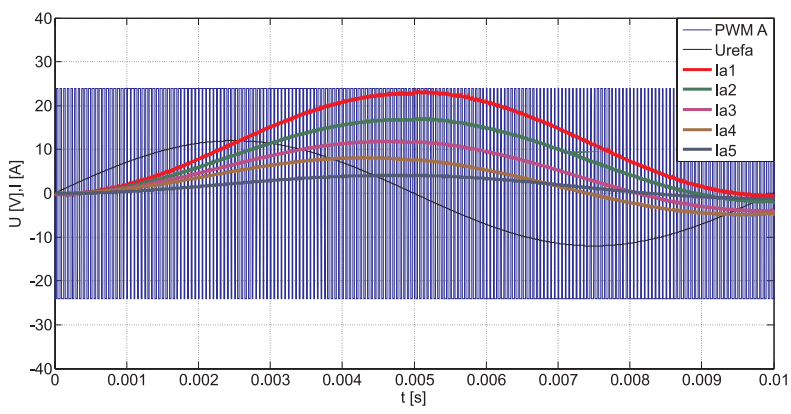

Fig. 10 Voltage of the half-bridge matrix converter and time waveforms of transient currents for $L / R=0.17 \mathrm{~s}, 0.04 \mathrm{~s}, 0.01 \mathrm{~s}, 52 \mathrm{~ms}, 14 \mathrm{~ms}$

\section{Conclusions}

The complex Fourier transformation was considered for threeand two phase orthogonal systems of converter output voltages, strongly non-harmonic ones. The solution given in the paper makes it possible to analyse more exactly the effect of each harmonic component comprised in the total waveform on resistive-inductive load or induction motor quantities. The proposed system with AC interlink in comparison with currently used conventional systems uses two single phase half bridge matrix converters with bipolar pulse-width modulation. The advantage is then less number of semiconductor devices of the converters. However, in practice, the necessary imposition of a dead-band, or blanking time, results in some distortion of the output voltage. Then, the dead-band, its symptoms and related remedies, are necessary to take into account for solutions.

\section{Acknowledgement}

The authors wish to thank for support to R\&D operational program Centre of excellence of power electronics systems and materials for their components No. OPVaV-2008/2.1/01-SORO, ITMS 26220120003 funded by European regional development fund (ERDF), and to VEGA Agency for the project No. 1/0470/09, and APVV project No-0510-06.

\section{References}

[1] SMITH, S.W.: The Complex Fourier Transform, The Scientist and Engineer's Guide to Digital Signal Processing, California Technical Publishing, 1997-2007, pp. 567-580, ISBN 0-9660176-3-3.

[2] HOFFMANN, M.: Fourier transform and DFT, Digital Signal Processing Maths, DESY, Hamburg, Germany, 2007, pp. 33-36; 42-56.

[3] CIZEK, V.: Discrete Fourier Transformation and Its Applications (in Czech), Mathematical Seminar Edition, SNTL Publisher, Prague (CZ), 1981. 
[4] BIRINGER, P. P., SLONIM, M.A.: Determination of Harmonics of Converter Current and/or Voltage Waveforms (New Method for Fourier Coefficient Calculations), IEEE Transactions on Industry Applications, Vol. IA-16, 2/April 1980, pp. 248-253.

[5] DEHBONEI, H., L. BORLE, NAYAR, C.V.: Optimal Voltage Harmonic Mitigation in Single-Phase Width Modulation, Proc. of AUPEC'01 Australasian Universities Power Engineering Conference, Perth (AUS), 2001, pp. 296.

[6] TAKEUCHI, T. J.: Theory of SCR Circuit and Application to Motor Control. Electrical Engineering. College Press, Tokyo (JP), 1968 - Russian Edition, Energija Publisher, Leningrad-St. Petersburg (RF), 1973, pp. 248, T 051(01)-73 130-73.

[7] DOBRUCKY, B., SUL, R., BENOVA, M.: Mathematical Modelling of Two-Stage Frequency Converter using Special Methods: Complex Conjugated Magnitudes and Orthogonal Park/Clarke Transformation Ones, J. of Applied Mathematics, Vol. II, 3/2009, ISSN 1337-6365.

[8] JARDAN, R. J., DEVAN, B. S., SLEMON, R. G.: General Analysis of Three Phase Inverters, IEEE Trans. on Industry and General Applications, 6/1969, pp. 672-679.

[9] JARDAN, R. J.: Modes of Operation of Three Phase Inverters, IEEE Trans. on Industry and General Applications, 6/1969, pp. 680-685.

[10] SOLIK, I., VITTEK, J., DOBRUCKY, B.: Time-Optimal Analysis of Characteristic Values of Periodical Waveforms in Complex Domain, J. of Modelling, Simulation, and Control, AMSE Press, Vol. A 28, 3/1990, pp. 49-64.

[11] MOHAN, N., UNDELAND, T. M., ROBBINS, W. P.: Power Electronics: Converters, Applications, and Design, John Wiley \& Sons, Inc., ISBN 0-471-42908-2.

[12] ZASKALICKY, P., ZASKALICKA, M.: Calculation of the Current Waveform of an AC Converter by Fourier series, Proc. of ISEM'05 Int'l Symposium on Electrical Machines, Prague, 2005, pp.194-199.

[13] ZASKALICKY, P., ZASKALICKA, M.: Mathematical Model of the Single-Phase Inverter with Pulse-Width Modulation, Proc. of Mechatronika'09 Int'l Conf., Tren. Teplice (SK), 2009, pp. CD-ROM.

[14] BENOVA, M., DOBRUCKY, B., SZYCHTA, E., PRAZENICA, M.: Modelling and Simulation of HF Half-Bridge Matrix Converter System in Frequency Domain, Logistyka (PL), No. 6/2009, ISSN 1231-5478. 